\title{
METODOLOGÍA PARA EL DISEÑO DEL MÉTODO DE EXPLOTACIÓN DE ARENAS SILÍCEAS MEDIANTE EL USO DE SOFTWARE ESPECIALIZADO
}

\author{
(Methodology for the design of the method of siliceous sandstones operation using special software)
}

\author{
Luis Ángel Lara González*, César Augusto Cubides Medina**, Edgar Rodrigo Hernández Buitrago*** \\ *Grupo de investigación GEAM, Universidad Pedagógica y Tecnológica de Colombia (UPTC) \\ Escuela de Ingeniería de Minas. luisangel.lara@uptc.edu.co \\ **Ingeniero en Minas, investigador grupo de investigación GEAM. cesarcubides2000@hotmail.com \\ ***Ingeniero en Minas, investigador Asesorías Mineras y Ambientales SAS. rodrigohernandezb10@gmail.com
}

(Recibido: 5 de Agosto de 2014 y aceptado 1 de Octubre de 2014)

\section{Resumen:}

Se reporta la metodología utilizada para el diseño del método de explotación de areniscas por bancos escalonados descendentes utilizando herramientas de software especializado. Los datos analizados fueron recolectados en campo para la licencia de explotación 14816 en el municipio de Melgar, departamento del Tolima. La caracterización del macizo rocoso se realizó a partir de pruebas físicas y mecánicas, sobre probetas cilíndricas, con el fin de obtener el valor de la resistencia máxima y el módulo de elasticidad de la roca. El rumbo y buzamiento del paquete de areniscas se determinó mediante proyección estereográfica utilizando el software DISP ${ }^{\circ}$. El factor de seguridad de los taludes se obtuvo con SLIDE®, se establecieron bancos de 8 metros de altura por 8 metros de ancho, con un ángulo de inclinación de $60^{\circ}$, lo cual generó un factor de seguridad de 2.1. El diseño del método de explotación fue hecho con GEOVIA SURPAC $®$, en una fase inicial de desarrollo en forma ascendente hasta alcanzar el nivel 11 de la explotación, para luego iniciar la explotación en forma descendente, para controlar la estabilidad de los taludes. Los resultados obtenidos permiten establecer una metodología general, para la elaboración de proyectos tendientes a optimizar el proceso de evaluación y selección del método de explotación mediante el uso de herramientas especializadas de diseño.

Palabras clave: diseño minero, software especializado, factor de seguridad, areniscas, Surpac.

\section{Abstract:}

The methodologies used for the design of the method of sandstones exploitation by descending staggered banks using specialized software tools are reported. The data analyzed were collected in the field for the operating license 14816 in Melgar, Tolima. The characterization of the rock mass was held from physical and mechanical tests, performed on cylindrical test tubes in order to obtain the value of the maximum strength and elastic modulus of the rock. The direction and dip of the sandstone package was determined by using the stereographic projection whit DISP $\AA$ software, and the safety factor of the slope was obtained with established banks whit SLIDE®. The slops are 8 meters high and 8 meters wide with a tilt angle $60^{\circ}$, which generated a safety factor of 2.1. The design of the mining method was carried out with GEOVIA SURPAC $®$, at an early stage of development ascending to the level 11 of the exploitation, to then start mining in descending order to control the stability of slopes. The results obtained allow a general methodology for the development of projects to optimize the process of evaluation and selection of mining method by using specialized design tools.

\section{INTRODUCCIÓN}

En Colombia son diversos los usos asociados a las arenas silíceas, los cuales en su gran mayoría están relacionados con el sector de la construcción y la industria del vidrio. Por tal razón, el número de minas y canteras de este tipo en el territorio nacional es elevado; tan solo en el año 2010 las solicitudes de títulos mineros para materiales de construcción fue de 1752 (Castillo, García \& Ardila, 2013), lo cual manifiesta un incremento significativo desde el año 2005, a la par con el de la construcción, que en el último año ascendió del 3,7 \% al 4,2 \% (Burgos, Gallardo \& Silva, 2014). Lo cual significa un aporte de un $6.4 \%$ del PIB nacional, después de la agricultura con $7.6 \%$, seguido por el de minas y canteras, que aportó un $4.3 \%$ del total general (Ardila, Nacionales, Victoria \& Acevedo, 2013).
Se sabe que en el país existen normas que regulan la explotación minera y que establecen los requisitos en cuanto a regalías, medio ambiente, planeación e impacto social, y que dichas normas son de estricto cumplimiento (Zapata \& Pizarro, 2012), con el fin de mitigar la explotación ilícita de los recursos minerales.

Teniendo en cuenta lo anterior, se propone establecer una metodología que permita al empresario minero y su cuerpo técnico, desarrollar el método de explotación de este tipo de minerales de una forma técnica, ambiental y económicamente sustentable, con el cumplimiento de todos los requerimientos ante las autoridades competentes. 
El diseño minero asistido por computador surge como una alternativa para cumplir con estos requerimientos, por eso en la actualidad son muchas las empresas y los proyectos que utilizan herramientas de software especializado en minería como el DISP ${ }$, SLIDE ${ }^{\circ}$, ROCLAB $1.0 \circledR$ y GEOVIA SURPAC $®$, en el diseño de sus operaciones cotidianas de exploración, preparación y explotación de diversos minerales (Grenon \& Laflamme, 2011; Kasmaee, Gholamnejad, Yarahmadi \& Mojtahedzadeh, 2010; Liu et al., 2012)

El modelamiento geológico en 3D y el diseño minero de superficie a cielo abierto, han permitido evaluar mediante métodos geoestadísticos los diferentes potenciales en variados depósitos minerales alrededor del mundo $\mathrm{y}$, a su vez, planear adecuadamente las operaciones mineras, convirtiendo a GEOVIA SURPAC $®$, en uno de los paquetes computacionales más populares (Haldar, 2013a, 2013b; Liu et al., 2012; Wang \& Huang, 2012; Wang et al., 2011).

Considerando lo anteriormente expuesto, el presente trabajo reporta la metodología utilizada para el diseño del método de explotación de areniscas por bancos escalonados descendentes utilizando herramientas de software especializado.

Los datos analizados fueron recolectados en campo para la licencia de explotación 14816 en el municipio de Melgar, departamento del Tolima, y analizados mediante pruebas físicas y mecánicas.

Con la información obtenida se establecieron los parámetros y variables de diseño, los cuales alimentaron los paquetes computacionales, a fin de seleccionar y diseñar el método de explotación que se adecuara a las condiciones del terreno, y que garantizara la viabilidad técnica, ambiental y económica del proyecto.

\section{MATERIALES Y MÉTODOS}

La zona de estudio se localiza en la vereda La Siberia, jurisdicción del municipio de Melgar. La principal vía de acceso es la carretera que de Carmen de Apicalá conduce al municipio de Cunday. A la altura del km 11 se desprende a la izquierda un carreteable que lleva directamente al área de la licencia.

El área objeto de contrato de concesión No. 14816 está delimitada por cuatro puntos de coordenadas (ver Tabla 1).

Tabla 1. Coordenadas de la licencia 14816

\begin{tabular}{|cccc|cc|}
\hline PUNTO & RUMBO & LONGITUD & \multicolumn{2}{|c|}{ COORDENADA } \\
\cline { 5 - 6 } & & $(\mathbf{m})$ & ESTE & NORTE \\
P.A & & - & - & 933496.1865 & 945777.3253 \\
& & & & \\
P.A & 1 & $67^{\circ}$ & 2053.1450 & 931600.000 & 944990.000 \\
$\mathbf{1}$ & 2 & 0 & 500 & 931600.000 & 945490.000 \\
$\mathbf{2}$ & 3 & $90^{\circ}$ & 1000 & 932600.000 & 945490.000 \\
$\mathbf{3}$ & 4 & $180^{\circ}$ & 500 & 932600.000 & 944990.000 \\
$\mathbf{4}$ & 1 & $270^{\circ}$ & 1000 & 931600.000 & 944990.000 \\
\hline
\end{tabular}

Las muestras de arenisca se tomaron del depósito correspondiente en su totalidad a la formación tabla, constituida principalmente por un banco de arenisca conglomerática masiva de grano fino a grueso, estratificada con un espesor aproximado de $91 \mathrm{~m}$, con un $96.46 \%$ de $\mathrm{SiO} 2$.
Las muestras de arenisca fueron taladradas y se obtuvieron probetas según la norma ASTM (C39, 2010).

Los análisis de resistencia mecánica se hicieron en un banco de compresión simple CONTROLS, con una célula de carga de $1500 \mathrm{KN}$ y en un banco de carga por punta Controls con célula de carga de $50 \mathrm{KN}$. Las pruebas de desgaste se realizaron en un cilindro marca Baldor a $500 \mathrm{rpm}$, durante 15 minutos.

Para definir el comportamiento del macizo rocoso, se requiere conocer los parámetros de cohesión y ángulo de fricción interna. Para ello se utilizó la aplicación ROCLAB1.0 ®, software de uso frecuente en estudios geotécnicos, que permite determinar los parámetros de resistencia del macizo rocoso. De acuerdo con el criterio de ruptura generalizado de Hoek-Brown Brown, el software siempre calcula los parámetros equivalentes de Mohr-Coulomb (c y ø) del macizo rocoso que, en este caso, son los parámetros utilizados para determinar el factor de seguridad de los taludes.

Los resultados obtenidos fueron ingresados al software DISP $®$, con el fin de analizar las discontinuidades del macizo rocoso mediante el método de proyección estereográfica, $\mathrm{y}$, de esta forma, calcular el factor de seguridad con SLIDE®.

El diseño de las labores de explotación y el cálculo de reservas, se realizaron mediante el uso de la aplicación GEOVIA

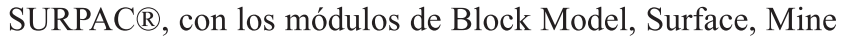
Desing y Geology Database.

\section{RESULTADOS Y DISCUSIÓN}

\subsection{Cálculo de reservas}

La evaluación cuantitativa del depósito es un factor importante en el diseño de un método de explotación, ya que brinda la oportunidad de calcular la duración del proyecto y las posibles utilidades económicas, de acuerdo con la oferta y demanda del producto final.

Las toneladas de arena silícea y los metros cúbicos de estériles presentes en la zona, se determinaron con el fin de definir la relación de descapote, la isobata límite de explotación y la relación de descapote límite.

Para el cálculo de reservas se utilizaron dos métodos. El primero es el método de perfiles: se obtienen bloques individuales de material, se efectúa el cálculo, se promedia el área entre dos perfiles consecutivos y se multiplica el valor obtenido por la distancia entre éstos; de esta manera se consigue un volumen que al ser multiplicado por el peso específico, arroja el contenido, en toneladas de arenisca, existente entre los dos perfiles evaluados.

Las reservas totales se consiguen al multiplicar las toneladas encontradas en los bloques individuales por un porcentaje de recuperación, que depende de la situación entre los dos perfiles, las condiciones topográficas del área, las condiciones geológicas de la zona y el diseño de la pared final para la explotación.
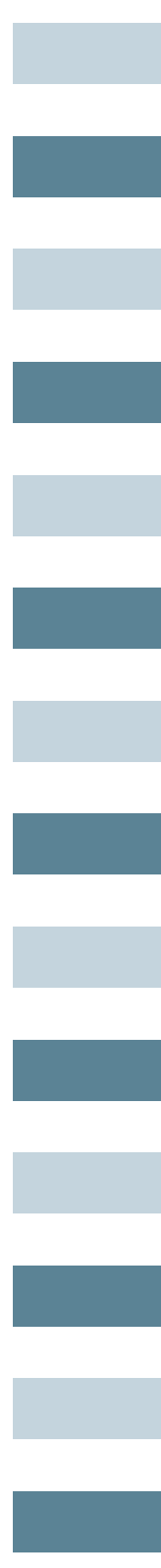

| 
Tabla 2. Cálculo de reservas de arenisca para la licencia 14816

\begin{tabular}{|lr|}
\hline \multicolumn{1}{|c|}{ TOTAL RECURSOS ESTIMADOS } \\
SEGÚN PERFILES \\
TOPOGRÁFICOS (m3)
\end{tabular}

Como se aprecia en la tabla, los recursos mineros explotables son de $10.717538,23$ metros cúbicos.

El segundo método utilizado fue el de intersección de superficies (DTM'S), mediante el cual se genera un modelo tridimensional del área a extraer o modelo de bloques, el cual fue levantado con GEOVIA SURPAC ${ }^{\circledR}$. Para el cálculo de reservas se obtiene la cantidad de material que se puede extraer a partir de la diferencia de cotas y de las intersecciones entre el bloque y la topografía; el software nos indica el volumen de mineral presente en el área de explotación (Figura 1).

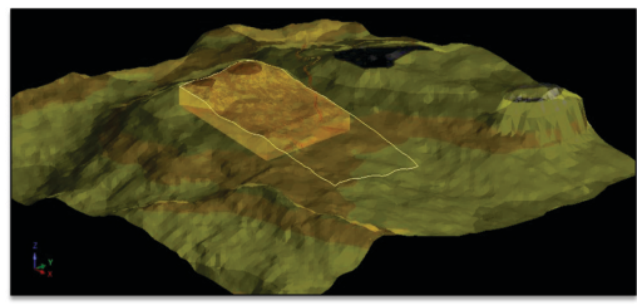

Figura 1. Modelo de bloque de arenisca, obtenido por el método de superposición de superficies

El volumen de mineral calculado por el método de intersección de superficies fue de 10.575.232 metros cúbicos, es decir, que difiere tan solo en un $2.53 \%$; lo cual le da confiabilidad a los resultados.

Según los resultados anteriores, las reservas mineras son las reservas explotables, menos las pérdidas generadas por el diseño y las labores de preminado y minado, además de restricciones como el área de la licencia. Por esta razón, se asumen en un $40 \%$, considerando el nivel de recuperación que se obtenga a partir del método de explotación implementado. Para el caso de la mina, este cálculo nos arroja un resultado de 26.793.846,580 toneladas de mineral explotable.

\subsection{Estudio geotécnico del macizo rocoso}

3.2.1 Propiedades físicas. El estudio geotécnico del macizo rocoso permitió determinar las propiedades físicas y mecánicas de la roca, información que es de gran importancia para el diseño de los taludes, en el que se tendrán en cuenta aspectos importantes como la densidad de diaclasas, buzamiento y la orientación de las juntas.

Para establecer las propiedades y variables necesarias para alimentar el software especializado, se determinaron, en primera instancia, las propiedades físicas de la arenisca presente en el depósito. Estas se obtuvieron en laboratorio mediante el ensayo de muestras extraídas directamente del macizo rocoso por un muestreo sistemático realizado sobre la extensión de la zona de estudio (Tabla 3 ).

3.2.2 Análisis granulométrico. Posteriormente se hizo el análisis granulométrico de las muestras. La proporción de los granos se determinó por medio de tamizado a diferentes tamaños de malla $(40,80,100$ y 200$)$; se obtuvo la relación entre la masa retenida en los diferentes tamices y la masa total de la muestra y la curva granulométrica para el depósito.
Según la curva, se determinó que predomina el tamaño de grano fino con diámetro entre 0,12 y $0,25 \mathrm{~mm}$ con un porcentaje de arena del $77,017 \%$, lo que nos indica que es una arena fina de tipo industrial.

\begin{tabular}{|c|c|c|}
\hline DESCRIPCIÓN & PROPIEDAD FÍSICA & $\begin{array}{l}\text { VALOR } \\
\text { PROMEDIO }\end{array}$ \\
\hline Arenisca & $\begin{array}{l}\text { Densidad total de la muestra } \\
(p) \mathrm{g} / \mathrm{cc}\end{array}$ & 2.23 \\
\hline Arenisca & $\begin{array}{l}\text { Densidad seca de la muestra } \\
(p \mathrm{~d}) \mathrm{g} / \mathrm{cc}\end{array}$ & 2.2 \\
\hline Arenisca & $\begin{array}{l}\text { Densidad saturada de la } \\
\text { muestra }(p \text { sat) } \mathrm{g} / \mathrm{cc}\end{array}$ & 2.56 \\
\hline Arenisca & $\begin{array}{l}\text { Porcentaje de humedad } \\
\text { (W) } \%\end{array}$ & 1.67 \\
\hline Arenisca & $\begin{array}{l}\text { Porcentaje de saturación } \\
\text { (S) \% }\end{array}$ & 8.07 \\
\hline Arenisca & $\begin{array}{l}\text { Porosidad } \\
\text { (n) } \%\end{array}$ & 36.59 \\
\hline Arenisca & $\begin{array}{l}\text { Relación de vacíos } \\
\text { (e) }\end{array}$ & 0.0576 \\
\hline Arenisca & $\begin{array}{l}\text { Peso unitario natural } \\
\text { (y) }\left(\mathrm{KN} / \mathrm{m}^{3}\right.\end{array}$ & 21.98 \\
\hline Arenisca & $\begin{array}{l}\text { Peso unitario seco } \\
\text { (yd) } \mathrm{KN} / \mathrm{m}^{3}\end{array}$ & 21.65 \\
\hline Arenisca & $\begin{array}{l}\text { Peso unitario saturado } \\
\text { (ysat) } \mathrm{KN} / \mathrm{m}^{3}\end{array}$ & 25.26 \\
\hline
\end{tabular}

3.2.3 Propiedades mecánicas. Una vez conseguidos los datos físicos de las muestras, se procedió a efectuar el análisis de las propiedades mecánicas de la arenisca, puesto que estos resultados son de vital importancia para el cálculo de los taludes y la determinación del factor de seguridad de los mismos. Para establecer estas propiedades, se realizó el ensayo de compresión simple y de carga por punta a 4 probetas cilíndricas de roca, para así obtener el valor de la resistencia máxima y el módulo de elasticidad de la misma. (Ver Figura 2 y Tabla 4)

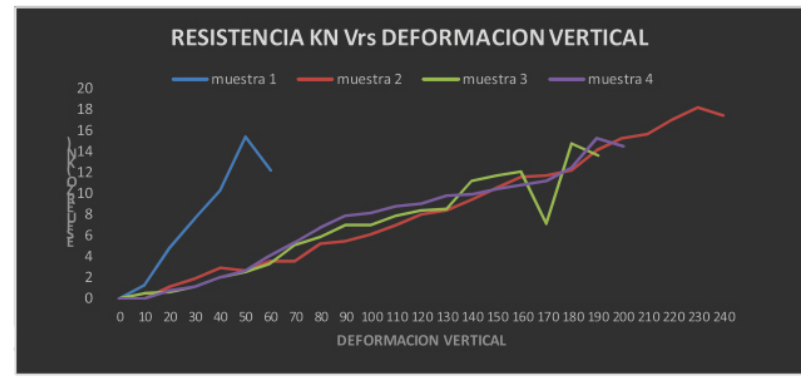

Figura 2. Gráfica esfuerzo-deformación, para muestras de arenisca

\begin{tabular}{|c|c|c|c|c|c|c|}
\hline & $\begin{array}{c}\text { Diámetro } \\
(\mathrm{mm})\end{array}$ & $\begin{array}{c}\text { Longitud } \\
(\mathrm{mm})\end{array}$ & $\begin{array}{c}\text { Peso } \\
\text { (g) }\end{array}$ & $\begin{array}{c}\text { Área } \\
\left(\mathrm{mm}^{2}\right)\end{array}$ & $\begin{array}{c}\sigma \\
(\mathbf{M p})\end{array}$ & $\begin{array}{c}\sigma \\
\left(K N / \mathrm{cm}^{2}\right)\end{array}$ \\
\hline 1 & 47 & 112 & 425.87 & 2628 & 4.6 & 0.46423 \\
\hline 2 & 55 & 105 & 415.65 & 2695 & 6.4 & 0.64564 \\
\hline 3 & 55 & 102 & 418.55 & 2750 & 4.9 & 0.49090 \\
\hline 4 & 54 & 102 & 418.55 & 2310 & 6.2 & 0.62770 \\
\hline \multicolumn{5}{|c|}{ Promedio Esfuerzos } & 5.52 & 0.55711 \\
\hline
\end{tabular}

3.2.4 Análisis de discontinuidades. El análisis de discontinuidades se hizo mediante el levantamiento de diaclasas en la extensión del paquete de arenisca, con el fin de determinar su rumbo, buzamiento, rugosidad, abertura, relleno y filtraciones.

Para la representación de dichos datos estructurales (fallas, diaclasas, estratos, etc.), se usan técnicas de proyección estereográfica. Este trabajo se apoya en el software DISP $®$.

Al ingresar los datos, se genera una base de datos con la orientación e inclinación del diaclasamiento presente en el macizo. 
Esto se hace para identificar las zonas donde hay mayor concentración de discontinuidades, y así determinar las familias que pueden generar cuñas y que tendrán incidencia en el macizo, lo que permitirá establecer la orientación de los taludes, a fin de mejorar las condiciones de seguridad.

En la Figura 3, se puede apreciar que existe una familia mayor, que contiene la concentración más alta de polos $(32.58 \%)$, y tres familias menores que oscilan en un rango de $11.9 \%$ y 28.5 $\%$.

Los buzamientos y direcciones predominantes se relacionan en el cuadro de orientaciones (Tabla 5).

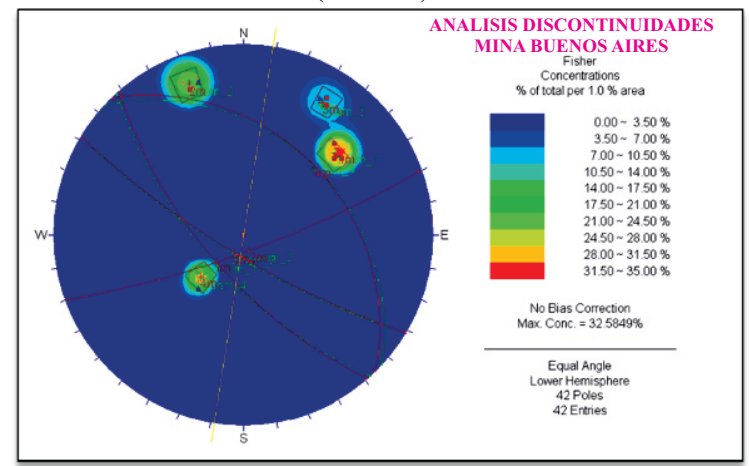

Figura 3. Diagrama de concertación de polos y ubicación de planos principales

Tabla 5. Distribución y orientación de los planos principales

\begin{tabular}{|ccccccc|}
\hline \multicolumn{2}{|l}{ Familias principales } & & & & & \\
\hline Id & $\begin{array}{c}\text { Juntas } \\
\left(\mathrm{m}^{3}\right)\end{array}$ & $\mathbf{N}^{\circ}$ Polos & Concentración & Buzamiento & Dirección Bz. & Rumbo \\
Familia 1 & 5 & 14 & $32.58 \%$ & $66^{\circ} \mathrm{SW}$ & $228^{\circ}$ & $\mathrm{N} 22^{\circ} \mathrm{W}$ \\
& & & & & & \\
Familia 2 & 4 & 10 & $23.8 \%$ & $80^{\circ} \mathrm{SE}$ & $160^{\circ}$ & $\mathrm{N} 70^{\circ} \mathrm{E}$ \\
Fami lia 3 & 2 & 5 & $11.9 \%$ & $77^{\circ} \mathrm{SW}$ & $211^{\circ}$ & $\mathrm{N} 60^{\circ} \mathrm{W}$ \\
Familia 4 & 4 & 12 & $28.5 \%$ & $35^{\circ} \mathrm{NE}$ & $43^{\circ}$ & $\mathrm{N} 50^{\circ} \mathrm{W}$ \\
\hline
\end{tabular}

Este análisis estereográfico es de vital importancia para la estabilidad del macizo rocoso, convirtiéndose en el punto de partida para determinar la orientación más conveniente de los taludes en el proyecto minero.

3.2.5 Clasificación del macizo rocoso. En las primeras etapas de todo proyecto minero, cuando la información del macizo rocoso es escasa o insuficiente, se hace necesario utilizar procedimientos técnicos que permitan establecer una idea del comportamiento y estabilidad del mismo, de allí la importancia de su caracterización y clasificación.

Los sistemas más populares son el de Bieniawski (Bieniawski et al., 1979) y Barton (Barton, Lien \& Lunde, 1974). Para el presente trabajo en particular se optó por la clasificación propuesta por Bieniawski.

La resistencia de la roca inalterada se obtuvo a partir de los datos de laboratorio (Tabla 6). El ensayo se aplicó a 6 probetas representativas del macizo, lo que dio como resultado el promedio aritmético del esfuerzo de la carga sobre las muestras, que es de $0,360320 \mathrm{KN} / \mathrm{cm} 2$ equivalente a 3,60 Mpa.

Tabla 6. Resultados del ensayo de carga por punta
\begin{tabular}{|ccc|}
\hline Mues. & $\sigma_{\mathrm{c} 1}\left(\mathbf{K N} / \mathbf{c m}^{\mathbf{2}}\right)$ & $\begin{array}{c}\sigma_{\mathrm{c} 2} \\
\left(\mathbf{K f} / \mathbf{c m}^{2}\right)\end{array}$ \\
\hline $\mathbf{1}$ & 0.275416 & 0.98339 \\
$\mathbf{2}$ & 0.336384 & 1.22915 \\
$\mathbf{3}$ & 0.444880 & 1.50304 \\
$\mathbf{4}$ & 0.235271 & 0.77506 \\
$\mathbf{5}$ & 0.484452 & 1.58926 \\
$\mathbf{6}$ & 0.385520 & 1.32487 \\
Promedio & $\mathbf{0 . 3 6 0 3 2 0}$ & $\mathbf{1 . 2 3 4 1 2}$ \\
\hline
\end{tabular}

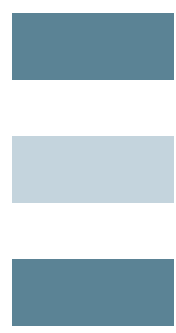

En este proyecto no fue posible realizar barrenos de exploración, por lo tanto el RQD se estima a partir del número de discontinuidades por unidad de volumen $(\mathrm{Jv})$, cuando estas son visibles en la superficie, por lo tanto:

$$
\mathrm{RQD}=115-3.3 \mathrm{JV}
$$

A partir de la inspección realizada en campo en un frente representativo, se estimó un (Jv), el cual corresponde a 18 diaclasa por metro cúbico (Tabla 9). De lo anterior resulta:

$$
\mathrm{RQD}=115-3.3 *(18)=55.6 \%
$$

Según la valuación obtenida a partir de la clasificación CSIR, se define como un macizo rocoso de calidad media, por lo tanto, las condiciones para realizar trabajos mineros son aceptables.

3.2.6 Análisis de deslizamientos. La estabilidad y la orientación de los taludes se basan en el análisis estereográfico de las discontinuidades presentes en el macizo rocoso, ya que de estas se extraen las orientaciones de las juntas, las cuales determinarán la posible formación de cuñas en las caras de los mismos. Este análisis se realizó como una fase de prefactibilidad o de estudios preliminares.

Para determinar la orientación más conveniente de los taludes, se analizan los criterios de intervalos de riesgos, los cuales se resumen a continuación:

Un intervalo angular de por lo menos \pm 15 grados en relación con el rumbo del talud.

Un intervalo angular en la línea de mayor pendiente del talud comprendido entre el circulo ø (ángulo de fricción interna) y unos $5^{\circ}$ adentro del arco meridional del talud

Un intervalo angular en la línea de mayor pendiente del talud de por lo menos $20^{\circ}$, comprendido entre las lecturas estereográficas $70^{\circ}$ y $90^{\circ}$, al lado opuesto al del arco meridional del talud.

Sombrear la media luna definida por el círculo ø y el arco meridional del talud.

Con este análisis y los resultados obtenidos en laboratorio para rocas, se determinó que los taludes deben tener una orientación principal de $\mathrm{N} 10^{\circ} \mathrm{W}$, con una variación de $\pm 30^{\circ}$, para los taludes de trabajo de este proyecto. 


\subsection{Diseño de la explotación}

En el caso del área en estudio, existe evidencia de la forma y proximidad del yacimiento a superficie, lo que facilita de alguna manera su extracción. Razón por la cual se requiere que la misma se realice de forma superficial, clasificando la mina dentro del sector de canteras, por tratarse de un material de depósito.

Teniendo en cuenta lo anteriormente expuesto, se debe hacer una evaluación para seleccionar el método de explotación adecuado para este tipo de mineral. Para tal fin, se deben tener en cuenta los parámetros relacionados con la forma del depósito, el relieve del terreno original, la proximidad a superficie, la inclinación, la complejidad y número de mineralizaciones, la distribución de la calidad del mineral en el depósito y el tipo de roca dominante.

Para el caso del presente trabajo, se encontró que la arenisca objeto de análisis es de origen sedimentario, de tipo concordante, ya que presenta una distribución armónica y homogénea, con rumbo y buzamiento bien definidos, y aflora en toda la extensión del área de concesión. Las labores de descapote de la capa vegetal son mínimas, ya que esta es de un espesor muy bajo, y labores de remoción de estéril no se realizarán.

Superficialmente, el terreno presenta una pendiente moderada hacia el $\mathrm{E}$, con un rumbo N32E del bloque de arenisca, el cual buza hacia el $36 \mathrm{SE}$. Con base en observaciones de campo realizadas, el bloque de mineral se comporta como una unidad litológica, que presenta un banco con espesores entre los 90 y $100 \mathrm{~m}$, con una distribución uniforme de la calidad en toda su extensión.

Evaluando parámetros como la favorabilidad de la topografía, la geología y los volúmenes de material que se va a extraer, la mina se ubica en un sistema de explotación a cielo abierto enmarcada por el método de canteras en ladera.

Se estimaron las siguientes opciones para el diseño del método de explotación por cantera: método de explotación por banco único, método de explotación por bancos escalonados descendentes. Este último se escogió como el ideal para el diseño de la explotación.

Teniendo en cuenta la información obtenida en campo y los resultados de laboratorio, se determinó que la primera fase de la explotación sería de desarrollo ejecutándose de forma ascendente, para garantizar el acceso de personal y maquinaria a los frentes de explotación en la cantera.

Para el diseño de la explotación, se utilizó el software GEOVIA SURPAC $\AA$, considerando los parámetros de diseño obtenidos mediante ensayos de laboratorio y los resultados de los software especializados mencionados anteriormente (Tabla 6).

Atendiendo la información de campo y la topografía del terreno, se diseñaron terrazas con una altura de banco de 8 metros, un ancho de banco de 10 metros, con un ángulo de talud de $60^{\circ}$.

Los taludes de trabajo se diseñaron con un ángulo de $29^{\circ}$, el ancho de la berma se calculó según el criterio de Ritchie (1963), modificadas por Hungr, Evans y Hazzard (1999).
$\mathrm{W}=0.2 \mathrm{H}+2.0$, para $\mathrm{H} \leq 9.0 \mathrm{~m}$

$\mathrm{W}=0.2 \mathrm{H}+4.5$, para $\mathrm{H}>9.0 \mathrm{~m}$

$\mathrm{H}=$ altura de banco $(\mathrm{m})$

Para una altura de $8 \mathrm{~m}$, el ancho de la berma será mínimo de 3.6 $\mathrm{m}$, sin embargo para el presente trabajo se establecieron bermas de $10 \mathrm{~m}$, con el fin de mejorar la estabilidad y movilidad de la maquinaria, además de favorecer las condiciones de restauración.

Tabla 6. Parámetros geométricos asociados al diseño

\begin{tabular}{|c|c|}
\hline Parámetro & scripción \\
\hline Tipo de depósito & Sedimentario - Concordante \\
\hline Rumbo yacimiento & $\mathrm{N} 32^{\circ} \mathrm{E}$ \\
\hline $\begin{array}{l}\text { Buzamiento } \\
\text { yacimiento }\end{array}$ & $36^{\circ} \mathrm{SE}$ \\
\hline $\begin{array}{l}\text { Espesor del } \\
\text { yacimiento }\end{array}$ & $91 \mathrm{~m}$ \\
\hline $\begin{array}{l}\text { Proximidad a } \\
\text { superficie }\end{array}$ & $\begin{array}{l}\text { Aflora en toda el área de } \\
\text { concesión }\end{array}$ \\
\hline Pendiente del terreno & $\begin{array}{l}\text { Moderada hacia el SE - Entre } 20 \\
\text { y } 25 \%\end{array}$ \\
\hline $\begin{array}{l}\text { Producción } \\
\text { requerida }\end{array}$ & $48 \mathrm{~m}^{3} /$ día - Aproximadamente \\
\hline $\begin{array}{l}\text { Límites de la } \\
\text { concesión }\end{array}$ & $\begin{array}{l}\text { Polígono rectangular-Área } \\
\text { superficial } 50 \mathrm{Ha} .\end{array}$ \\
\hline
\end{tabular}

Con los parámetros de diseño establecidos se define un ángulo final de $35^{\circ}$, el cual está acorde con el dimensionamiento geométrico de la explotación.

Con esta información se procedió a calcular el factor de seguridad para el talud final y los taludes de trabajo, a fin de verificar si los parámetros de diseño generan condiciones favorables para dar inicio al proyecto minero.

Para determinar los parámetros de entrada con el fin de calcular el factor de seguridad (FS), se utilizó el software ROCKLAB ${ }^{\circledR}$, de uso frecuente en estudios geotécnicos, que permite determinar los parámetros de resistencia del macizo rocoso, de acuerdo con el criterio de ruptura generalizado de Hoek y Brown (1997). Los datos de salida suministrados por este paquete (ver Tabla 7), permiten alimentar el programa SLIDE ${ }^{\circledR}$ de Rocscience para la determinación del factor de seguridad (FS). Aquí se hace una simulación del talud con cargas distribuidas que establecen el esfuerzo en las caras del talud visualizando las zonas críticas generadas por la interacción de maquinaria, equipos y las propiedades geotécnicas del suelo. Para ello se utilizaron dos métodos de análisis del talud, por una parte el método de Bishop y Morgenstern (1960).

Tabla 7. Parámetros geométricos asociados al diseño

\begin{tabular}{|lccc|}
\hline \multicolumn{2}{|l|}{ Datos de entrada ROCLAB } & \multicolumn{2}{c|}{ Datos de salida ROCLAB } \\
\hline sigci & $5.52 \mathrm{Mpa}$ & $\mathrm{m}_{\mathrm{b}}$ & 0.564 \\
GSI & 55.6 & $\mathrm{~s}$ & 0.0001 \\
$\mathbf{m}_{\mathbf{i}}$ & 9 & $\mathrm{a}$ & 0.513 \\
$\mathbf{D}$ & 0.7 & $\mathrm{c}$ & $0.208 \mathrm{Mpa}$ \\
$\boldsymbol{V}$ & $22.3 \mathrm{KN} / \mathrm{m}^{3}$ & $\varnothing$ & $24.67^{\circ}$ \\
$\mathbf{H}$ & $8 \mathrm{~m}$ & $\mathrm{E}_{\mathrm{m}}$ & $2108.06 \mathrm{Mpa}$ \\
\hline
\end{tabular}

En la Tabla 8 se puede observar la variación que tiene el factor de seguridad según los parámetros anteriormente mencionados.

Por tal razón se establecen bancos de explotación de $8 \mathrm{~m}$ de altura, con bermas para el diseño del talud final de $8 \mathrm{~m}$ de ancho. Durante el avance se utilizan bancos de trabajo de $10 \mathrm{~m}$ de ancho, para otorgarle un mayor espacio de movilidad a la maquinaria, con ángulos de talud de $60^{\circ}$, según las condiciones del frente de explotación, para dejarlos con el ángulo definitivo de $60^{\circ}$. Condiciones que proporcionan un factor de seguridad de 2.1 , lo cual mejora en gran medida la operación de la maquinaria en la mina. 
Tabla 8. Variación del FS en función de la altura y ángulo del talud

\begin{tabular}{|ccccc|}
\hline & FS & \multicolumn{3}{c|}{ Ángulo del talud } \\
\hline & & $50^{\circ}$ & $60^{\circ}$ & $70^{\circ}$ \\
& & & & \\
\hline & & & & \\
\hline
\end{tabular}

Para el diseño de las terrazas y los bancos de trabajo se utilizó el modulo Mine Desing de GEOVIA SURPAC $®$ y el módulo de intersección de superficies. La Figura 4 muestra el diseño de la fase de desarrollo, donde se pueden apreciar las vías en forma ascendente siguiendo el contorno topográfico, y realizadas simultáneamente con las labores de desmonte de la capa vegetal.

El inicio de la explotación tendrá lugar en la cota $920.96 \mathrm{msnm}$, hasta alcanzar los 1008.96 msnm, que será el límite superior de la explotación, a partir de allí se dará inicio a la segunda fase de diseño y de explotación, mediante el método de bancos escalonados descendentes (Figura 5).

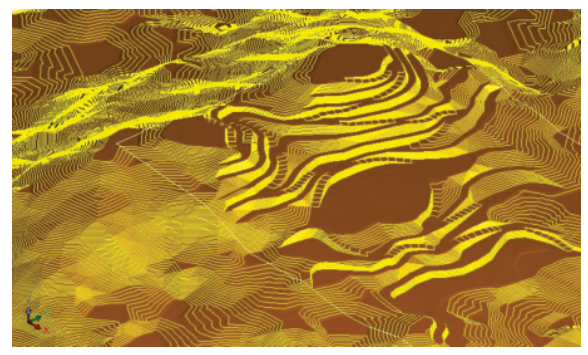

Figura 4. Labores de desarrollo Fase 1 del proyecto.

Para iniciar la explotación, como plantea el método seleccionado, es necesario contar con la mina desarrollada y preparada, a fin de realizar el terraceo en forma descendente, el cual irá dejando los taludes finales de acuerdo con el diseño proyectado con bermas de $8 \mathrm{~m}$, alturas de banco de $8 \mathrm{~m}$ y ángulo de talud de $60^{\circ}$, según los cálculos hechos para los taludes.

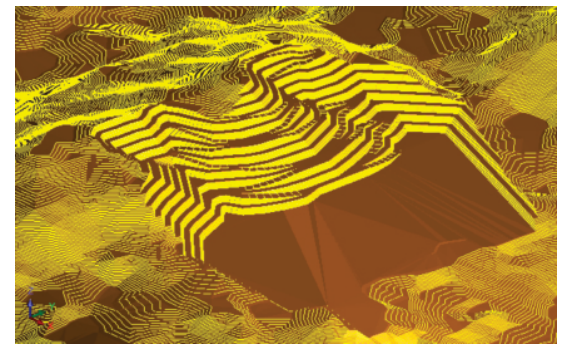

Figura 5. Labores de explotación Fase II del proyecto.

\subsection{Usos y aplicaciones}

Debido a la demanda que tiene este material en la industria refractaria colombiana así como en la construcción, y, a su vez, por las características del yacimiento presente en esta zona y por la variedad de granulometrías, lo convierten en un mineral idóneo para diferentes aplicaciones industriales, ya que tiene la ventaja de ser explotado en canteras, lo cual facilita su extracción y comercialización al venderse. Como se extrae directamente o posterior al tamizado, y se comercializa la arena clasificada, esto le da un valor agregado al producto.

\section{CONCLUSIONES}

El estudio geomecánico del macizo rocoso de la explotación para la licencia 14816, según el sistema de valoración RMR, determinó que este macizo es de clase III; si bien es de tipo medio, presenta condiciones estructurales favorables para las operaciones mineras.

Las características geomorfológicas y la distribución uniforme del depósito en toda la extensión del área de trabajo, favoreció la concepción de un diseño de explotación que cuenta con todas las condiciones técnicas para su ejecución.

Las condiciones de estabilidad de los taludes son favorables, esto se pudo constar con la ayuda del programa SLIDE® de Rocscience, el cual define un factor de seguridad aceptable de 2.1 en todos los casos analizados. Por esta razón se optó por establecer bancos de $8 \mathrm{~m}$ de altura, bermas de $10 \mathrm{~m}$ de ancho y taludes con $60^{\circ}$ de inclinación, dimensiones que favorecieran tanto la restauración ambiental, como las condiciones de seguridad para el personal y la maquinaría.

Se estableció una metodología para seleccionar y diseñar el método de explotación para depósitos de arenisca.

El desarrollo de la mina se hará en forma ascendente en una primera fase, hasta alcanzar el nivel 11. A partir de allí, la explotación será de forma descendente por el método de bancos escalonados descendentes, ya que este permite tener un mejor control de la estabilidad de los taludes.

Se apoyó el diseño y la selección del método de explotación mediante el uso de software especializado en minería, el cual se convierte en una herramienta fundamental y de uso obligatorio para la elaboración de este tipo de proyectos.

\section{REFERENCIAS}

Ardila, D. S., Nacionales, C., Victoria, A. \& Acevedo, V. (2013). Cuentas Nacionales Trimestrales Producto Interno Bruto, 4-9.

Barton, N., Lien, R. \& Lunde, J. (1974). Engineering classification of rock masses for the design of tunnel support. Rock Mechanics, 6(4), 189-236.

Bieniawski, Z. T. et al. (1979). The geomechanics classification in rock engineering applications. In 4th ISRM Congress.

Bishop, A. W. \& Morgenstern, N. (1960). Stability coefficients for earth slopes. Geotechnique, 10(4), 129-153.

Burgos, K. O., Fernando, C., Gallardo, R. \& Silva, G. (2014). Hacia la consolidación del sector edificador en Colombia. Avances del año 2013 y desafios para el 2014, (54), 1-18.

C39, A. (2010). Standard Test Method for Compressive Strength of Cylindrical Concrete Specimens.
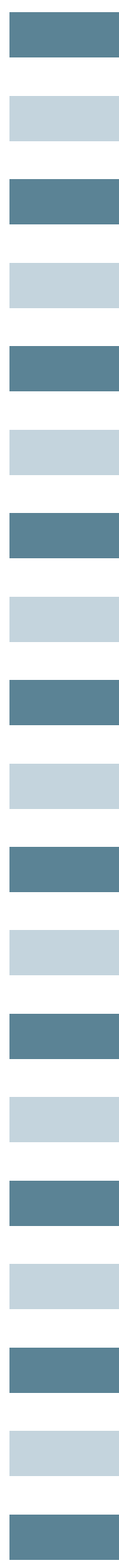
Castillo, A. M., García, J. \& Ardila, R. C. V. B. (2013). Anuario estadístico minero colombiano. Bogotá: Ministerio de Minas y Energia

Grenon, M. \& Laflamme, A.-J. (2011). Slope orientation assessment for open-pit mines, using GIS-based algorithms. Computers \& Geosciences, 37(9), 1413-1424. doi:10.1016/j.cageo.2010.12.006

Haldar, S. K. (2013a). Mineral Exploration. In Mineral Exploration (pp. 287-322). Elsevier. doi:10.1016/B978-0-12416005-7.00015-5

Haldar, S. K. (2013b). Mineral Exploration. In Mineral Exploration (pp. 193-222).Elsevier. doi:10.1016/B978-0-12416005-7.00011-8

Hoek, E. \& Brown, E. T. (1997). Practical estimates of rock mass strength. International Journal of Rock Mechanics and Mining Sciences, 34(8), 1165-1186.

Hungr, O., Evans, S. G. \& Hazzard, J. (1999). Magnitude and frequency of rock falls and rock slides along the main transportation corridors of southwestern British Columbia. Canadian Geotechnical Journal, 36(2), 224-238.
Kasmaee, S., Gholamnejad, J., Yarahmadi, A. \& Mojtahedzadeh, H. (2010). Reserve estimation of the high phosphorous stockpile at the Choghart iron mine of Iran using geostatistical modeling. Mining Science and Technology (China), 20(6), 855-860. doi:10.1016/S1674-5264(09)60295-7

Liu, X., Luo, Z., Yang, B., Lu, G., Cao, S. \& Jiang, X. (2012). Visible calculation of mining index based on stope 3D surveying and block modeling. International Journal of Mining Science and Technology, 22 (2), $139-144$. doi:10.1016/j.ijmst.2011.08.001

Ritchie, A. M. (1963). Evaluation of rockfall and its control. Highway Research Record, (17).

Wang, G. \& Huang, L. (2012). 3D geological modeling for mineral resource assessment of the Tongshan $\mathrm{Cu}$ deposit, Heilongjiang Province, China. Geoscience Frontiers, 3(4), 483-491. doi:10.1016/j.gsf.2011.12.012

Wang, G., Zhang, S., Yan, C., Song, Y., Sun, Y., Li, D. \& Xu, F. (2011). Mineral potential targeting and resource assessment based on 3D geological modeling in Luanchuan region, China. Computers \& Geosciences, 37(12), 1976-1988. doi:10.1016/j.cageo.2011.05.007

Zapata, M. E. \& Pizarro, A. P. (2012). Casos Valle del Cauca (río Dagua)-Chocó (río San Juan). 